\title{
La política monetaria y el crecimiento económico: la tasa de interés de referencia del Banco de México
}

Monetary policy and economic growth:The reduction of the interest rate

Reference Bank of Mexico

\section{Resumen}

A partir que el banco central asume de manera explícita a la tasa de interés como instrumento dominante de la política monetaria y adopta el control de la inflación como objetivo central, se argumenta que las variaciones de la tasa de interés del banco no pueden ampliar la demanda ni acercar la demanda efectivo al producto potencial ni generar crecimiento económico.

En el contexto del sistema bancario mexicano menores tasas de referencia no reducen el resto de las tasa de interés, ni amplían el crédito. En relación al último anuncio del Banco de México de reducir la tasa referencia, en este trabajo se argumenta que su propósito es disminuir el costo de la deuda del gobierno y abaratar el costo de los créditos que el banco central otorga a los bancos comerciales.

\section{Palabras clave:}

- Política Monetaria

- Bancos Centrales y sus políticas

- Crecimiento económico
Noemi Levy Orlik*

\begin{abstract}
Since central banks explicitly consider the rate of interest as their main monetary policy instrument and inflationary targets have been adopted as the most important target we argue that central bank reference rates reduction doesn't expand effective demand and it's unrelated to economic growth.

In the Mexican banking system a reduction of the central "main" interest rates does not reduce credit costs nor amplify the credit volumes. In relation to Banco de Mexico last announcement of decreasing its "reference" rate we assume that it was aimed to lower government debt costs so as central bank credits costs issued to commercial banks.
\end{abstract}

\section{Keywords:}

- Monetary Policy

- Central Banks and their Policies

- Economic Growth

JEL: E52, E58, F43

\section{Introducción}

En 6 junio del 2014, por onceava ocasión consecutiva el Banco de México determinó bajar la tasa de referencia en cincuenta puntos bases, al reducir la tasa interbancaria objetivo de un día de 3.5 a 3\%. Ello se realizó en el contexto de la revisión hacia la baja, por parte del banco central, de la tasa de crecimiento de la economía mexicana.

Desde la perspectiva de la autoridad central, éste cambio de previsiones de la tasa de crecimiento de la economía abrió un espacio para relajar la política monetaria. Reiteradamente, el Banco de México ha señalado que el bajo desempeño de la economía mexicana se debe al reducido mercado interno, señaladamente de la inversión productiva doméstica y del consumo privado,

* Profesora de tiempo de completo de la Facultad de Economía de la UNAM. Este trabajo fue realizado en el marco del proyecto PAPIIT IN 303314. . - . 
Economía Informa núm. 387 julio - agosto • 2014 " "

se añade que está decisión no tendrá impacto en la inflación actual ni en la esperada, y tampoco en la relación del peso con respecto al dólar (BDM, 2014).

A propósito de esta decisión de política económica, en este trabajo nos proponemos a realizar un análisis crítico sobre las bases que esgrimió el Banco de México para tomar tal decisión. Se postula que las reducciones de la tasas de referencia apuntan a objetivos distintos del crecimiento económico, los cuales, por ejemplo, pueden reducir el costo de préstamos del banco central a los bancos comerciales, o bajar los costos de captación del gobierno, vía emisiones de bonos.

Nuestros argumentos se dividen en cinco secciones. En la segunda, se discuten las bases teóricas de la dominación de la tasa de interés como el principal mecanismo de transmisión de política económica que cuestiona la visión teórica tradicional más simple. En la siguiente (tercera) se discute la evolución de la política monetaria del Banco de México a la luz del cambio de instrumentos de la política monetaria y las razones que se esgrimen para modificar las tasas de interés. En la cuarta sección se analiza el movimiento de la tasa de interés, el endeudamiento de los principales agentes y actividades económicas, así como su impacto en el crecimiento económico. Finalmente, en la quinta sección se exponen las principales conclusiones.

\section{Las tasas de interés y la teoria económica: antecedentes teóricos de la visión del Banco Central}

La vieja discusión al interior de la corriente teórica dominante de que si los bancos centrales controlan la base monetaria y, a través del multiplicador monetario a la masa monetaria y, de esa manera estabilizan los precios, o sólo pueden determinar la tasa de interés de corto plazo, obligándose acomodar la demanda de reservas de la banca comercial y, por esa vía acotar el crecimiento económico y mantener controlada la inflación, pareciera haber sido saldado en los años noventa del siglo pasado. Ello puede resumirse en la respuesta que Blinder (1998) ofreció, ante el reclamo de teóricos monetarista, que los banqueros centrales abandonaron los agregados monetarios. Señaló: "nosotros no abandonamos los agregados monetarios, ellos nos abandonaron a nosotros" (ibid, p. 28), aclarando que en periodos de grandes movimientos de capital sólo es posible controlar la tasa de interés que puede resumirse de la siguiente manera: cuando las perturbaciones de la Is (ahorro-inversión, aclaración nuestra) son grandes, es mejor elegir la oferta monetaria ...La feroz inestabilidad de la curva LM estimadas en Estados Unidos, Reino Unido y muchos otros 
países, que comenzó en la década de 1970 y se ha mantenido hasta la actualidad, llevó a los economistas y a los responsables de las políticas económicas a extraer la conclusión de que la elección de la oferta monetaria como objetivo, sencillamente no es una opción viable (Ibid, p. 27).

Ello implica que la visión fisheriana-marshaliana más simple, contenida en la ecuación de la teoría rudimentaria del dinero (Harris, 1981) se volvió una visión anticuada y pasada de moda, llegándose a señalar que los bancos centrales nunca pretendieron controlar la base monetaria (Bindseil, 2004). En este contexto, se acepta un planteamiento de amplio consenso que, incluso, fue parte de la teoría económica dominante en las primeras décadas del siglo pasado, donde se señalaba que el dinero es endógeno y que el banco central sólo puede determinar la tasa de interés (Véase Chick, 2005).

Obviamente, el gran disenso se centró sobre las formas en qué el dinero afecta al sector productivo y financiero. Pareciera que la teoría dominante está sustentada en la idea que el dinero únicamente puede modificar los precios. Este mito fue derribado por Toporowski $(2005,2014)$ quien al revisar los planteamientos teóricos de varios economistas influyentes de finales del siglo XIX e inicios del siglo Xx (que agrupo en la categoría de teorías criticas del financiamiento, los cuales, en algunos casos sirvieron como base del monetarismo, las expectativas racionales, el neokeynesianismo y el nuevo consenso clásico) muestra que diferenciaron entre la masa monetaria y los créditos, con diferentes impactos sobre la economía real (véase Hawtrey ${ }^{1}$ ), y que el dinero podía ser inestable y generar ciclos de negocios (donde también está incluido Irving Fisher por sus famoso artículo The Debt Deflation Theory of Great Depressions, publicado en 1933. Ello indicaría que el dinero puede modificar el curso de la actividad económica.

El antecedente de esta discusión se encuentra en la escuela monetaria alemana-austriaca, que sentó las bases del dinero endógeno relacionado con los ciclos de negocios, los cuales, supuso que eran acotados (Véase Wicksell, 1907). Desde esta perspectiva, los bancos centrales, vía variaciones de la tasa de interés monetaria, acomodan la liquidez que demandan los bancos comerciales. Específicamente, sobre la base del planteamiento de Wicksell, quién sostuvo que si la tasa de interés mercado es superior a la tasa de interés 'natural', se genera ahorro

1 De acuerdo a Hawtrey (Toporowski, capítulo IV, 2014) la expansión de los créditos, por parte de la banca, no está limitada por ningún factor real y, a su vez, generar ciclos económicos con auges y depresiones. Este autor se aleja de la noción de equilibrio, dominante en el análisis monetariofinanciero, incluso, en planteamientos tan novedosos como los de Keynes (especialmente en la Teoría General). A su vez adopta la noción de una tasa de interés monetaria y que la demanda determina la oferta, específicamente en el ámbito creditico,Véase Toporowski, 2014. 
Economía Informa núm. 387 julio - agosto • 2014 - " " " " " " " " " -

'forzado', baja la producción y decaen los precios. Ello genera una reducción de la demanda crediticia y suben las reservas del banco central. Con base en el supuesto de equilibrio entre la tasa de interés de mercado y la 'natural', el banco central reduce la tasa de interés de mercado (que se supone un instrumento de política monetaria), Si ésta tasa se sitúa por debajo de la tasa de interés 'natural', aumenta la demanda de los créditos, se expande la demanda de bienes y servicios e inversión y suben los precios. Estas variaciones se ven reflejadas en la reducción de las reservas del banco central y ello se convierte en una señal para que aumente la tasa de interés de mercado, generándose un ciclo económico acotado en torno a la tasa "natural", que iguala la inversión y el ahorro, garantizado mediante el supuesto de equilibrio entre la tasa de interés de mercado (que determina la banca central) y la tasa 'natural' (tasa de ganancia del capital productivo). Resaltamos que el canal de transmisión tiene lugar a través de procesos acumulativos de inflación (deflación) que modifican las reservas del banco central e indican la necesidad de modificar la tasa de interés de mercado.

Este planteamiento fue revivido por Taylor (1993), dando origen a la famosa 'regla de Taylor' (Véase Levy, 2010 a y una amplia literatura que contiene innumerables modelos que el banco central debe adoptar para determinar la tasa de interés de mercado, donde se incluyen otras objetivos intermedios, como el tipo de cambio (v.g., Hüfner, 2004).

La visión original, propuesta por Taylor supone que la decisión del banco central en torno a la tasa de interés de mercado está en función de la brechas del producto, la brecha de la inflación, dada una tasa de interés 'natural'. Los supuestos de este planteamiento son, primero, existe un nivel de ingreso potencial o 'natural' (distinto del pleno empleo) que no genera presiones inflacionarios, que se diferencian del producto observado, cuya brecha indica la presencia de capacidad instalada no utilizada, la cual puede cerrarse vía menores tasas de interés de mercado. Segundo, existe un nivel de precios de mercado que si se iguala a los precios "correctos" puede garantizar la igualdad entre la tasa de interés de mercado y la 'natural'. Entonces, si la actividad económica no está sujeta procesos inflacionarios, la política monetaria vía reducción de la tasa de interés puede incrementar la demanda, dada la capacidad potencial productiva existente, sin provocar presiones inflacionarias.

Las limitantes de esta visión son numerosas, en el marco de este trabajo destacamos dos. La primera está relacionada con el concepto de producto potencial, determinado por la NAIRU (tasa de desempleo no aceleradora de inflación, postulado por Modigliani y Papademos, 1975) que supone que el gasto (donde se incluye la inversión) no puede ampliar la capacidad ociosa, o sea, 
la inflación es resultado de presiones generadas por la demanda. Ello implica que el empleo está en función de la capacidad instalada que se encuentra fija, independientemente del volumen de recursos productivos ociosos, lo cual ha demostrado ser falso por la teoría de la demanda efectiva, porque el gasto de la inversión puede ampliar la capacidad instalada de una economía, sin generar inflación. ${ }^{2}$ Segundo, se argumenta que dada la tasa de interés 'natural' y un conjunto de precios 'correctos', expresados a través de una inflación 'objetivo', la tasa interés mercado se puede igualar a la tasa 'natural' y garantizar el máximo volumen de empleo no generador de inflación. Este supuesto es ampliamente criticado, entre otros, por Smithin (2007) debido al desconocimiento de la tasa natural, es imposible plantearse una inflación 'objetivo' y, por consiguiente, no se sostiene la regla de Taylor. En términos generales Lavoie (2004) caricaturiza este planteamiento como la de un 'viejo vino en nuevas barricas', para señalar que esta visión se sustenta en viejos planteamientos de la teoría económica, sin que los autores estén conscientes de los desarrollos teóricos anteriores, ni sus limitaciones.

\section{La Política Monetaria del Banco de México y los razonamientos para reducir la tasa de interés}

La primera parte de la discusión está relacionada al régimen de la política monetaria dominante en México en los últimos veinte años. Después de abandonar una política monetaria acomodaticia vinculada al financiamiento del déficit fiscal y canalización directa de recursos financieros a la producción (v.g., políticas de encaje legales, cajones crediticias, limites a la tasas de interés, entre otras, véase Mántey, 2010 y Levy, 2010 ) a la luz del nuevo ordenamiento financiero internacional y los paradigmas del modelo neoliberal financiarizado se modificaron los instrumentos y las bases de la política monetaria.

Específicamente, a partir del 1ero de abril de 1994 el Banco de México obtiene autonomía sobre sus decisiones de política monetaria, ${ }^{3}$ siendo una de sus principales distinciones acotar el objetivo de la política monetaria a un sólo

2 En el marco de los países que no operan con dinero internacional y sus cuentas comerciales externas no están equilibradas (i.e., tiene déficit comerciales) y requieren de insumos para producir, los flujos extranjeros para financiar dichos insumos, es una limitante del crecimiento. La limitación externa introduce el tipo de cambio como una variable central en la función de reacción del banco central (Hüfner, 2004). Ésta políticas, empero, ha generado agudas sobrevaluaciones de las monedas locales que limitan el crecimiento económico.

3 Mayores referencias, véase http://www.banxico.org.mx/disposiciones/marco-juridico/ley-delbanco-mexico.html 
Economía Informa núm. 387 julio - agosto • 2014 " "

resultado, que es la estabilidad de precios; eliminándose el propósito de lograr el crecimiento económico, en un contexto de estabilidad de precios. Es decir, a partir de 1994, en el marco de los acuerdos del Tratado de Libre Comercio con América del Norte (TLCAN), el Banco de México se adhiere al consenso económico internacional dominante, sustentado en la NAIRU y el combate a la inflación.

A la luz del supuesto que el banco central no puede modificar directamente la inflación se señala que debe adoptar objetivos operacionales, ${ }^{4}$ donde la tasa de interés, determinada por el banco central, tiene un papel central, existiendo dos variantes. Una, es la política de saldos de las cuentas bancarias en el banco central, y la otra es la determinación directa de la tasa de interés a un día.

La primera (saldos de las cuentas corrientes) entró en operación en 1995 (septiembre) mediante la imposición de un objetivo sobre las cuentas corrientes de la banca, sustentado en un esquema que "equivale a inyectar o retirar toda la liquidez necesaria, a tasas de mercado, para que las cuentas corrientes finalicen el periodo de medición en cero" (Véase Instrumentos de la Política Monetaria a través de un Objetivo Operacional de Tasas de interés, pp. 1 y 2). Se aclara: "Cuando el banco central desea mantener una política restrictiva anuncia un saldo objetivo negativo, y, para una política monetaria expansiva, un saldo objetivo positivo (Íbid). "Cuando existe un "corto" (saldo objetivo negativo) el banco central continúa inyectando toda la liquidez que el sistema necesita, sin embargo, una parte de ésta, el monto del corto, la provee a tasas de interés penales (actualmente dos veces la tasa de interés de fondeo interbancario a un día)" (Íbid, cursivas nuestras). Y, concluyen "Esta acción presiona las tasas de interés de mercado al alza pues los bancos buscan pedir prestados los fondos para evitar el pago de las tasas penales del banco central" (Ibid). Entonces, el Banco de México mediante el anuncio de los objetivos de los saldos corrientes podía afectar las tasas de interés de mercado, acomodando todas las reservas solicitadas por la banca comercial, aunque a diferentes precios.

En 2003 el Banco de México tránsito hacia la imposición directa de una tasa de interés. En este contexto, determinó saldos finales diarios en vez de saldos acumulados (Íbid, p. 3) acompañado de anuncios sobre "su postura de

4 Los objetivos operacionales son aquellas instrumentos que dispone el banco central que "afectan de manera directa a un grupo de variables nominales que, a su vez, tienen impacto sobre los determinantes de la inflación", véase Instrumentos de la Política Monetaria a través de un Objetivo Operacional de Tasas de Interés, http://www.banxico.org.mx/politica-monetaria-e-inflacion/material-de-referencia/intermedio/politica-monetaria/\%7B16DC84D2-A904-6373-28E329659E530FDB\%7D.pdf 
política monetaria en fechas predeterminadas" (Íbid). En abril del siguiente año (2004) complementó el "anuncio del nivel del "corto" con señalamientos más precisos sobre el nivel deseado de las 'condiciones monetarias' o las 'tasas de interés'. O sea, desde esa fecha “las tasas de interés de fondeo interbancario a un día se ajustaran en movimientos puntuales y estables... por lo que el mercado ha operado "de facto" siguiendo una tasa señalada por el Banco de México (Íbid, p. 4). Este documento señala que el último movimiento de las tasas de fondeo interbancario relacionado con un cambio en el "corto" fue en febrero de 2005. (Íbid, p. 5).

Formalmente, a partir del 21 de enero de 2008, el Banco de México adoptó como objetivo operacional la tasa de fondeo bancario a plazo de un día; aclarando que no se modificaron los instrumentos para lograr sus objetivos:

Las operaciones de mercado abierto tendrán como objetivo llevar a cero el saldo agregado de las cuentas corrientes de los bancos al final del día. El Banco de México continuará inyectando o retirando toda la liquidez faltante o sobrante del sistema a través de estas operaciones.

Las tasas a las que se remuneran excedentes en las cuentas corrientes o se cobran los sobregiros seguirán siendo de cero y de dos veces la tasa de fondeo bancario a plazo de un día, respectivamente (Íbid. p. 5).

El segundo elemento de ésta discusión es revisar los fundamentos del Banco de México para reducir la tasa de interés objetivo y los impactos que se esperan obtener a través de esta modificación, a la luz de la reducción de 50 puntos bases, en junio del 2014. Éste reducción fue antecedido por tres cambios en 2013 que redujeron la tasa objetivo de 4.5\% (monto determinado en 2008) a 3.5\%. Las consideraciones realizadas por los miembros de la Junta de Gobierno del Banco de México se basan en las expectativas internacionales de crecimiento, las condiciones financieras del mercado internacional, las políticas monetarias de los países avanzados, y sus efectos sobre México. Éstas fueron plasmadas en la "Minuta Número 28" de la Reunión de la Junta de Gobierno del Bando de México, con motivo de la decisión de política monetaria, anunciada el 6 de junio del 2014.

Teniendo en consideración que México es un país en desarrollo, emergente, dinamizado por las exportaciones con fuertes desequilibrios negativos en la cuenta externas (déficit comerciales) y se distingue por tener mercados financieros domésticos poco profundos, el tipo de cambio aparece como un objetivo intermedio fundamental, y opera como mecanismo de transferencia 
Economía Informa núm. 387 julio - agosto • 2014 " "

de la variación de precios externos a internos y, en conjunto con los rendimientos sobre los títulos financieros (tasa de interés) atrae o contrae flujos financieros externos, necesarios para equilibrar la cuenta externa.

Bajo estas condiciones, el informe económico sobre el resto del mundo (de la referida Minuta Número 28) resalta la reducción del crecimiento económico de Estados Unidos, por condiciones no económicas (climatológicas) cuyo efecto fue reducir la demanda de sus importaciones y se pronostica que se mantendrá la atonía económica de Europa. También se señala que no habrá presiones inflacionarias porque los precios de las materias primas y alimentos están a la baja, por consiguiente las presiones inflacionarias por el lado de la oferta estarán ausentes.

Por su parte, se prevé que la política estadounidense de estabilización monetaria $^{5}$ (principal socio comercial de México) no modificara las tasas de referencia de dicho país y no hay signos sobre la emergencia de importantes alteraciones del mercado financiero de los países desarrollados. Además las tasas de interés de los bonos gubernamentales de los países desarrollados disminuyeron "para alcanzar en algunos caso, los niveles más bajos, observados en el año” (Íbid, p. 5), con base en lo anterior se concluye que:

La política monetaria altamente acomodaticia en los principales países avanzados, que se han reflejado en un ambiente de muy baja volatilidad en los mercados financieros, ha favorecido el buen desempeño de activos de mayor riesgo. $\mathrm{Al}$ respecto, los diferenciales de las tasas de interés entre distintos instrumentos de renta fija y los bonos gubernamentales se encuentran cerca de los más bajos en los últimos 10 años. Adicionalmente los índices accionarios han registrado ganancias importantes y, en algunos casos, presentan valuaciones por encima de su promedio de largo plazo" (Íbid, p. 9).

Entonces, los países desarrollados han incrementado temporalmente la capacidad ociosa y, debido al pronostico que no habrá presiones inflaciones ni aumentara la incertidumbre, se amplía la brecha de precios. Tampoco se espera cambios en la reducción de los flujos internacionales, por el contrario, se prevé que podrían aumentar.

5 La política de normalización monetaria estadounidense es la reducción de la comprar de activos se ha mantenido relativamente inalterada sin alterar el rango de la tasa de objetivos de los fondos federales de dicha economía, Íbid, p. 4. 
Por su parte, la previsión de los mercados financieros de los países emergentes también presentan una evolución favorable, apoyada por una menor incertidumbre de la política monetaria de los países avanzados y, además se observa menores tensiones geopolíticas (hasta la reunión del 6 de junio del 2014). Ello ha dado lugar a una apreciación del tipo de cambio de sus monedas frente al dólar, presentándose también un mayor rendimiento potencial ajustado por riesgo, con resultados favorables en los instrumentos financieros de los mercados accionarios; y bajas tasas de interés de mediano y largo plazos. Se señala que éste "desempeño es producto, entre otros factores, del incremento de los flujos de inversión de cartera hacia los mercados emergentes por tercer mes consecutivo". O sea, se reitera que prevalecerán las condiciones para un amplio flujo de capitales externos hacia los países en desarrollo.

Respecto a la economía mexicana se destaca que la evolución del sector productivo tiene un entorno de bajo crecimiento económico, especialmente del gasto interno y señaladamente del gasto de inversión fija; o sea no se espera una ampliación de la capacidad productiva (aunque señalan que ha incrementado las importaciones de bienes de capital fijo); y se apunta que el gasto de consumo privado ha descendido. En consecuencia, por el lado de la economía domestica no hay presiones sobre la demanda y la desocupación se ubico "con niveles superiores a los observados a principios de año" (Íbid, p. 17); y añaden que los índices de productividad tiene una pendiente ascendente mientras los costos laborales presentan continua pendiente descendente (Íbid, véase grafica 49, p. 17). Al respecto se apunta: "Los incrementos en los principales indicadores de la economía continúan siendo moderados. Esto, junto con la tendencia que ha exhibido la productividad media del trabajo, condujo a que los costos unitarios continuaran disminuyendo" (Ibid).

Otro elemento importante es que no esperan presiones inflacionarias futuras. De hecho la inflación ha descendido (véase Cuadro 1: Índice Nacional del Precios al Consumidor, Ibid, p. 20) y se resalta que las principales presiones podrían provenir de los precios de los productos pecuarios y de las tarifas autorizadas por el gobierno. En un ambiente de globalización, con atonía en el crecimiento mundial, las primeras no deben generar incrementos de precios por la posibilidad de acceder a productos del resto del mundo; mientras que las segundas están sujetas a decisiones gubernamentales. 
Economía Informa núm. 387 julio - agosto • 2014 .

\begin{tabular}{|c|c|c|c|c|c|c|}
\hline \multicolumn{7}{|l|}{ Cuadro 1: Pairwise Granger Causality Test } \\
\hline \multicolumn{7}{|c|}{ Series en niveles, datos diarios } \\
\hline Rezagos & \multicolumn{3}{|c|}{1 Rezago (1 día) } & \multicolumn{3}{|c|}{2 Rezagos (2 dias) } \\
\hline Null Hypothesis: & Obs & F.Stat & Prob. & Obs & F.Stat. & Prob. \\
\hline CETE91 (1) does not Granger Cause CETE28 (1) & 1555 & 99.58 & 0.00 & 1554 & 45.06 & 0.00 \\
\hline CETE28 does not Granger Cause CETE91 & & 0.06 & 0.80 & & 2.50 & 0.08 \\
\hline OBjetivo (1) does not Granger Cause CETE28* & 1555 & 25.96 & 0.00 & 1554 & 9.11 & 0.00 \\
\hline CETE28 does not Granger Cause OBJETIVO* & & 30.35 & 0.00 & & 15.29 & 0.00 \\
\hline TIIE (2) does not Granger Cause CETE28 & 1555 & 41.12 & 0.00 & 1554 & 21.07 & 0.00 \\
\hline CETE28 does not Granger Cause TIIE ${ }^{\star}$ & & 27.88 & 0.00 & & 30.46 & 0.00 \\
\hline OBJETIVO does not Granger Cause CETE & 1555 & 4.48 & 0.03 & 1554 & 4.55 & 0.01 \\
\hline CETE91 does not Granger Cause OBJETIVO* & & 56.29 & 0.00 & & 28.35 & 0.00 \\
\hline TIIE does not Granger Cause CETE91 & 1555 & 1.23 & 0.27 & 1554 & 32.91 & $0.00^{\star}$ \\
\hline CETE91 does not Granger Cause TIIE ${ }^{\star}$ & & 109.64 & 0.00 & & 133.30 & 0.00 \\
\hline TIIE does not Granger Cause OBJETIVO^ & 1555 & 93.15 & 0.00 & 1554 & 52.53 & 0.00 \\
\hline OBJETIVO does not Granger Cause TIIE ${ }^{\star}$ & & 88.15 & 0.00 & & 777.52 & 0.00 \\
\hline \multicolumn{7}{|c|}{ Series en diferencias, datos diarios } \\
\hline Rezagos & \multicolumn{3}{|c|}{1 Rezago (1 día) } & \multicolumn{3}{|c|}{2 Rezagos (2 días) } \\
\hline Null Hypothesis: & Obs & F. Stat & Prob. & Obs & F. Stat & Prob. \\
\hline D(CETE91) does not Granger Cause D(CETE28)^ & 1554 & 20.23 & 0.00 & 1553 & 21.86 & 0.00 \\
\hline D(CETE28) does not Granger Cause D(CETE91) & & 4.94 & $0.023^{\star}$ & & 1.84 & 0.16 \\
\hline D(OBJETIVO) does not Granger Cause D(CETE28) & 1554 & 3.23 & 0.07 & 1553 & 13.33 & $0.00^{\star}$ \\
\hline D(CETE28) does not Granger Cause D(OBJETIVo) & & 0.33 & 0.56 & & 0.17 & 0.84 \\
\hline D(TIIE) does not Granger Cause D(CETE28)^ & 1554 & 15.88 & 0.00 & 1553 & 12.23 & 0.00 \\
\hline D(CETE28) does not Granger Cause D(TIIE)^ & & 44.09 & 0.00 & & 23.95 & 0.00 \\
\hline D(OBJETIVO) does not Granger Cause D(CETE91) & 1554 & 3.59 & 0.06 & 1553 & 18.72 & $0.00^{\star}$ \\
\hline D(CETE91) does not Granger Cause D(OBJETIVo) & & 0.53 & 0.47 & & 0.39 & 0.68 \\
\hline D(TIIE) does not Granger Cause D(CETE91)^ & 1554 & 65.37 & 0.00 & 1553 & 28.68 & 0.00 \\
\hline D(CETE91) does not Granger Cause D(TIIE) ${ }^{\star}$ & & 204.88 & 0.00 & & 102.99 & 0.00 \\
\hline D(TIIE) does not Granger Cause D(OBJETIVo) & 1554 & 7.38 & 0.01 & 1553 & 12.01 & $0.00 \star$ \\
\hline D(OBJETIVO) does not Granger Cause D(TIIE) ${ }^{\star}$ & & 1557.43 & 0.00 & & 780.76 & 0.00 \\
\hline
\end{tabular}




\section{Continuación}

\section{Cuadro 1: Pairwise Granger Causality Test}

Series en niveles, datos mensuales

\begin{tabular}{|c|c|c|c|c|c|c|}
\hline Rezagos & \multicolumn{3}{|c|}{1 Rezago (1 mes) } & \multicolumn{3}{|c|}{2 Rezagos (2 meses) } \\
\hline Null Hypothesis: & Obs & F.Stat & Prob. & Obs & F.Stat & Prob. \\
\hline CETES28 does not Granger Cause ACTIVA ${ }^{\star}$ & 239 & 27.03 & 0.00 & 238 & 6.49 & 0.00 \\
\hline ACTIVA does not Granger Cause CETES28^ & & 42.99 & 0.00 & & 14.47 & 0.00 \\
\hline CETES91 does not Granger Cause ACTIVA & 239 & 3.05 & 0.08 & 238 & 0.99 & 0.37 \\
\hline ACTIVA does not Granger Cause CETES91 & & 7.75 & $0.01 \star$ & & 2.34 & 0.10 \\
\hline CETES91 does not Granger Cause CETES28* & 239 & 32.12 & 0.00 & 238 & 14.18 & 0.00 \\
\hline CETES28 does not Granger Cause CETES91* & & 18.01 & 0.00 & & 6.56 & 0.00 \\
\hline
\end{tabular}

Tasa de interés interbancaria de equilibrio (TIIE)

TO:Tasa de fondeo bancario

(1) Tiene raiz unitaria orden uno

(2) Tiene raiz unitaria de orden uno pero la seria estacionaria

$\rightarrow$ causalidad; ... no causa

La causalidad de Granger evalúa una regresión auxiliar, con el fin de determinar si los errores de una variable, explican a la otra, definiéndose así la causalidad.

Si la probabilidad es mayor a $0.05 \%$, no se rechaza la hipótesis nula de que no existe causalidad.

Si la probabilidad asociada es menor a $0.05 \%$, se rechaza la hipótesis nula de que no existe causalidad, por tanto existe causalidad de Granger entre las series

Todas las pruebas se realizan al 95\% de confianza.

Fuente:

http://www.banxico.org.mx/estadisticas/index.html, Tasas de interés y precios de referencia en el mercado de valores

Con base en las previsiones ofrecidas por el Banco de México, los miembros de su Junta de Gobierno, deciden reducir la tasa de interés, con base en que: primero, la economía presenta una mayor holgura porque, por un lado se reducen la demanda externa (exportaciones mexicanas) por la previsión de una menor tasa de crecimiento a la esperado por parte del principal social comercial (principalmente porque no se recupera el sector inmobiliario que vía efecto riqueza expande el consumo), continuara fragilidad de la zona euro, y no se esperan grandes variaciones de los países emergentes exitosos (China) y, por otro, hay consenso que el gasto interno continuara deprimido. Incluso se menciona que el mercado laboral sigue mostrando gran holgura y que no se perciben presiones de demanda en el mercado crediticio.

Segundo, declaran que la decisión de reducir la tasa de interés de referencia se toma sobre el consenso que no esperan presiones inflacionarias del resto del mundo ni de la economía mexicana, por consiguiente no se prevén de la inflación observada. 
Economía Informa núm. 387 julio - agosto • 2014 " "

Tercero (y, quizá de manera más relevante) los flujos financieros a las economías emergentes y, especialmente a México (por presentar fundamentos macroeconómicos sólidos) no se verán alterados porque no habrá variaciones en las tasa de referencia de Estadios Unidos ${ }^{6}$ y de Europa no esperan crecientes niveles de volatilidad financiera (incluso se señala que se han reducido en los último meses, Ibid, p. 23). Sobre esta base la mayoría de los integrantes sostuvo que en el contexto de menor volatilidad en los mercados financieros internacionales, la búsqueda de rendimientos ha reactivado los flujos de capital hacia economías emergentes (p. 24). Uno de ellos explicó que entre las fortalezas con las que cuenta México para enfrentar una posible reversión de flujos de capital destacan su sólido marco macroeconómico, la flexibilidad en las tasas de interés y el tipo de cambio, su elevado nivel de reservas internacionales y la Línea de Crédito Flexible con el Fondo Monetario Internacional. Agregó que México pudo enfrentar adecuadamente la volatilidad registrada a partir de mayo del año pasado sin intervenciones en los mercados cambiarios o medidas extraordinarias (Ibid). Otro integrante puntualizó que, si bien estas fortalezas acotan los riesgos, es preciso tener presente que el grado de apertura de la economía mexicana acrecienta el impacto potencial de los movimientos de capitales, lo que subraya la importancia del manejo adecuado de la política monetaria, fiscal y de estabilidad financiera (Ibid, p, 25 y 26).

Resumiendo la conclusión se toma en con base en las siguientes premisas: "Primera, afirmaron que se ha observado una evolución de la actividad económica más débil que la anticipada, y que se ha ampliado la holgura en la economía, la cual se espera persista en el horizonte en el que opera la política monetaria. Segunda, insistieron que la inflación se ha comportado mejor que lo previsto, que no se han presentado efectos de segundo orden ante los cambios en precios relativos registrados a finales del año pasado y principios del presente, y que las expectativas de inflación se mantienen bien ancladas. Tercera, señalaron que el manejo ordenado de las finanzas públicas permite a su vez una mayor flexibilidad en el manejo de la política monetaria. Cuarta, añadieron que se ha retrasado el momento en el que se espera inicie el alza de tasas en Estados Unidos. Adicionalmente, algunos integrantes afirmaron

6 Sobre la previsiones de la tasa de interés de referencia de Estados Unidos, "un miembro señaló que la Reserva Federal ha sido clara en que el primer incremento en su tasa de referencia probablemente ocurra mucho después de terminado su programa de compra de activos y en que es de esperarse que la tasa de referencia permanezca en niveles menores a los considerados normales en el largo plazo por un tiempo relativamente prolongado, aun después de que el empleo y la inflación se encuentren en sus niveles objetivo" Ibid, p. 23 
que el mercado ya ha incorporado la posibilidad de menores tasas de interés nacionales, e hicieron notar que la tasa a la que se están colocando los Cetes en el mercado está por debajo de la actual tasa de referencia. Finalmente, un integrante enfatizó que una disminución en la tasa de referencia por parte del Banco de México es congruente con la consolidación del avance estructural en el control de la inflación (Ibid, 26-27).

\section{Los mecanismos de transmisión: la tasa de interés, el financiamiento y el crecimiento económico}

En este apartado se discute el comportamiento de la economía mexicana, observando la relación entre la tasa de interés objetivo y el resto de las tasas de interés; la evolución del crédito y su conexión con el gasto y el crecimiento económico, recordando que la economía mexicana está sustentada en una organización crediticia, con mercados de capitales angostos pequeños y poco profundos y acceso limitado de financiamiento para las empresas micro, pequeñas y medianas.

\section{Gráfica I}

\section{Evolución de las principales tasas de interés}

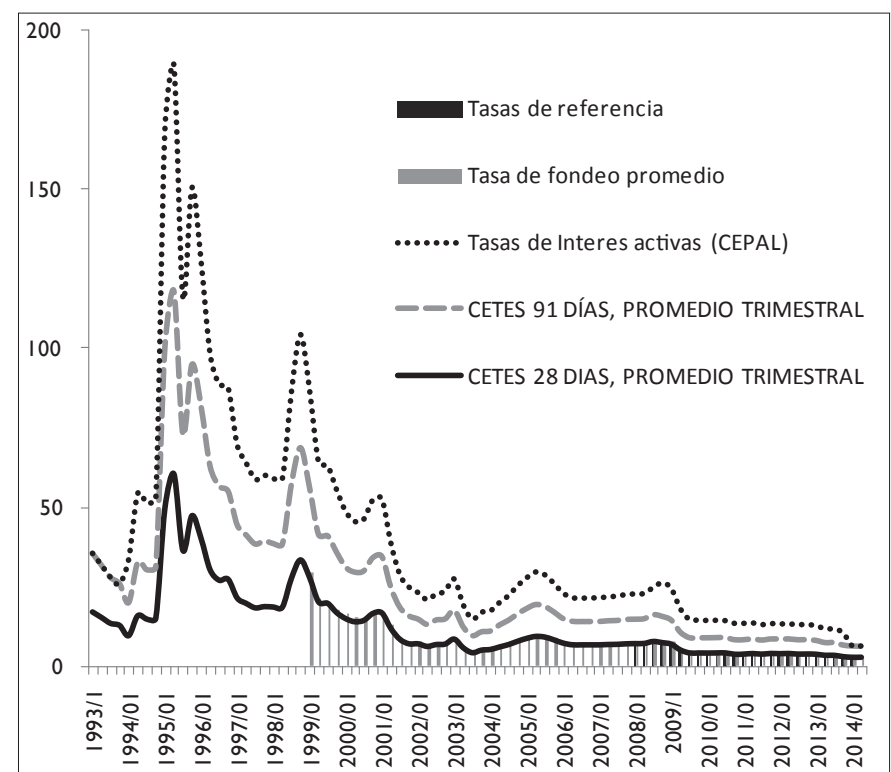

Fuente. elaboración propia con datos de Banco de Mexico y de CEPAL.

Disponible en: http://estadisticas.cepal.org/, Estadísticas e indicadores, Económicos, Sector financiero y monetario, Indicadores Financieros.

http://www.banxico.org.mx/estadisticas/index.html, Tasas de interés y precios de referencia en el mercado de valores. 
Economía Informa núm. 387 julio - agosto • 2014 - " " " " " " " " " -

Una primera observación es que la tasa de interés de fondeo, la tasa de Cetes a 28 y 91 días y la tasa de referencia se mueven de manera conjunta, con márgenes por las diferencias de temporalidad. La tasa de interés activa muestra variaciones muy similares a las anteriores tasas, incluida la tasa de referencia y de fondeo del banco central, con montos más elevados que dan cuenta de los riesgos incluye la actividad crediticia (véase gráfica 1).

Las pruebas de causalidad nos indican una direccionalidad desde la tasa Cetes de 91 a 28 días, lo cual es válido en series a niveles con un rezago de 1 y 2 días. Este resultado se validad en la matriz de correlaciones con datos en diferencia donde los CETES a 91 muestran comportamiento diferentes, incluso con signos negativos con respecto a los cetes Cetes de 29 días. ${ }^{7}$ Adicionalmente hay indicaciones de causalidad desde la tasa de Cetes a 91 días a la TIIE (tasa de interés interbancaria de equilibrio) con rezago a un día; y con respecto a la tasa objetivo ${ }^{8}$ (niveles y rezago de un día). Segundo, también hay sugerencias de causalidad desde la tasa objetivo hacia la tasa de Cetes de 28 día (niveles, rezago de un día, y diferencias de un día (con niveles de probabilidad de 90\%) y dos días) y, también hay causalidad de la tasa objetivo a la tasa de Cetes de 91 en diferencias con un y dos días. Finalmente, respecto a las tasas activa (solo se considera en niveles porque la tasa activa y la TIIE son estacionarios) indican una causalidad desde la tasa activa hacia la tasa de Cetes de 91 con un mes de rezago, véase cuadro 1. Lo anterior indica que la tasa de interés dominante de la economía es la tasa objetivo, así como la tasa de Cetes 91 días y a partir de dichas tasas se puede explicar el valor de otras.

Una segunda observación es que las tasas de interés activa por diferentes tipos de créditos (el Banco de México las denomina tasas implícitas) se mueven de manera coordinada, con excepción de la tasa de interés relacionada con las carteras de crédito del consumo y las tarjetas no bancarias. Específicamente, para el periodo de 2008-2013 encontramos que dicha tasa está por encima del promedio de la tasa implícita de la cartera total de créditos de la banca múltiple, y sus movimientos son diferentes a la tasa de interés de referencia, así como de las demás tasas, véase gráfica 2. Ello se debe a que dicho segmento crediticio opera en un mercado altamente concentrado, sin competencia de otros estratos del mercado financiero (véase Domínguez, 2011).

\footnotetext{
7 Este resultado se valida en la matriz de correlaciones con datos en diferencia donde la tasa de CETES a 91 muestran comportamiento diferentes (con signos negativos) con respecto a las demás tasas (Cetes 29 días, tasa objetivo, y TIIE).

8 Se hace referencia a la tasa de fondeo bancaria.
} 


\section{Gráfica 2}

\section{Evolución de la tasas implicitas (activas) de la banca múltiple por} tipo de cartera crediticia

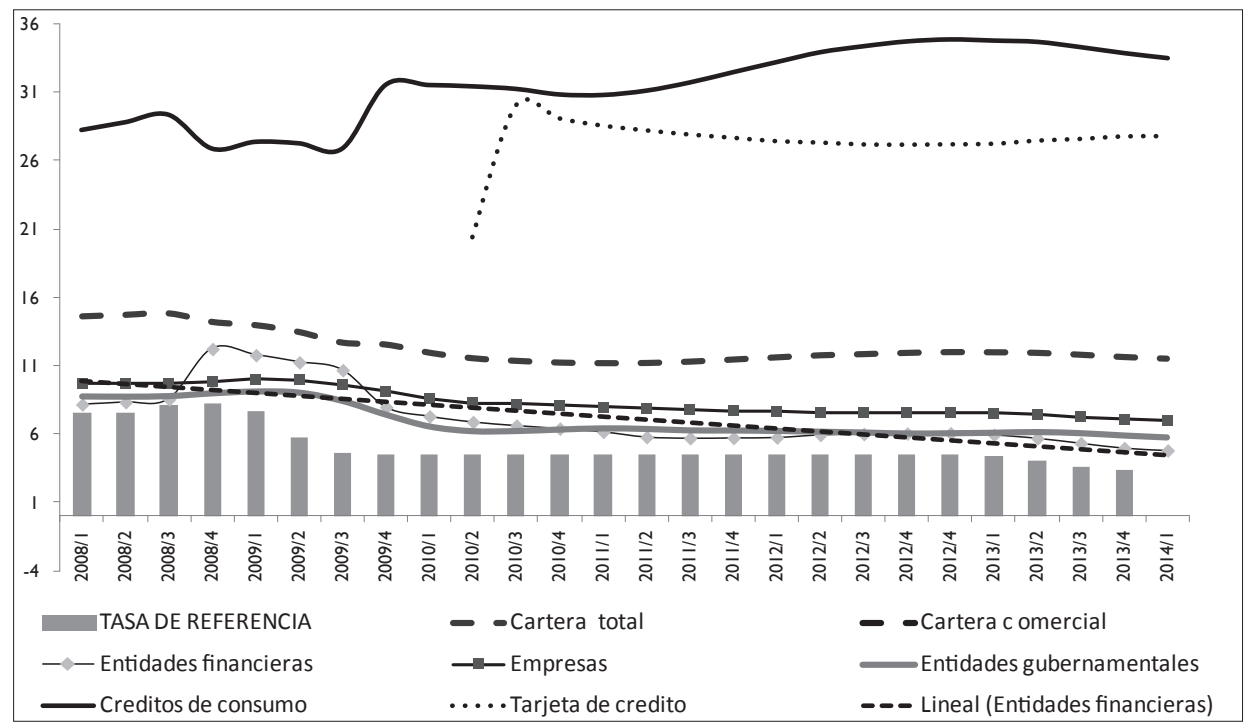

Fuente: elaboración propia con base en información de la Comisión Nacional Bancaria y deValores. Disponible en: http://portafoliodeinformacion.cnbv.gob.mx/, Banca Múltiple, Información de la situación financiera, Indicadores financieros: históricos (series desde diciembre 2000).

Un tercer elemento es que el movimiento de las tasas activas (o implícitas) no está muy correlacionado con el endeudamiento al sector privado no financiero. Se observa una caída del endeudamiento total al sector privado no financiero que se repone en la década del 2000 sin alcanzar los niveles de 1994. En diciembre de ese año alcanzó un máximo de $53 \%$ con respecto al producto; seguido por una tendencia descendente, cuyo mínimo se alcanza en el segundo trimestre del 2006, para posteriormente recuperarse, con niveles alrededor de $40 \%$, desde 2013 en adelante, véase gráfica 3. Adicionalmente, tiene lugar un cambio en la composición del endeudamiento, observándose una drástica caída del componente bancario. Al inicio del periodo, el crédito bancario fue de alrededor de $60 \%$ del endeudamiento total sector privado no financiero, bajo a un mínimo de 30\% en el último trimestre de 2005: con una leve recuperación posterior. La reducciones no parecen corresponder a los movimientos de las tasas de referencia ni de las tasa de interés restantes (véase gráfica 3). Por su parte el endeudamiento no bancario tiene un comportamiento inverso: se acelero entre 1995 y 2005 (alcanzando 70\% del total), posteriormente se reduce y, en los últimos años se estabiliza entre 60 y $65 \%$ (véase Gráfica 3). 
Economía Informa núm. 387 julio - agosto • 2014 " "

\section{Gráfica 3}

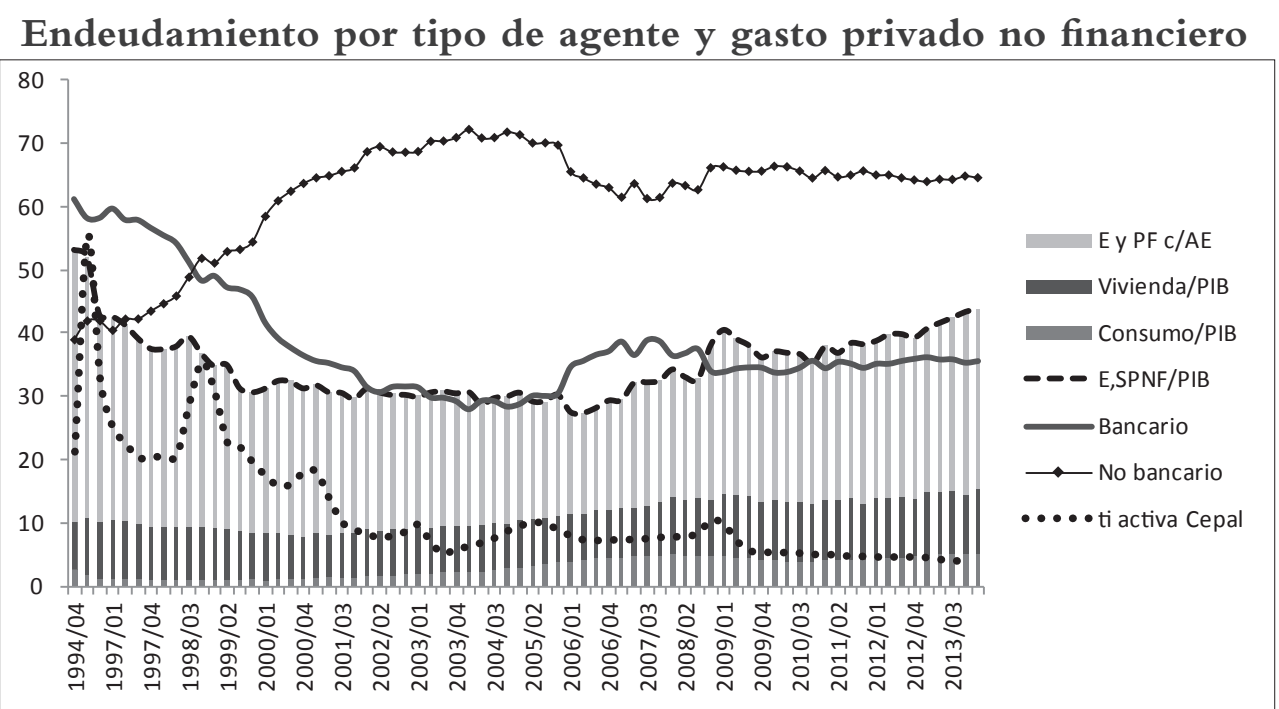

EyPE c/AE: empresas y personas fisicas con actividad empresarial

Fuente: elaboración propia con base en:

http://estadisticas.cepal.org/, Estadísticas e indicadores, Económicos, Sector financiero y monetario, Indicadores Financieros

http://www.banxico.org.mx/estadisticas/index.html, Financiamiento e información financiera de intermediarios financieros, Financiamiento al sec privado del país, bancario y otras fuentes principal (met 2003)

http://www.inegi.org.mx/sistemas/bie/, Producto interno bruto trimestral, base 2008 , Series originales, Valores a precios de 2008, Producto interno bruto, a precios de mercado (Periodicidad: Trimestral)

Se debe anotar que la caída del endeudamiento del sector privado no financiero se debe especialmente a la menor deuda de las empresas y las personas físicas con actividad empresarial (véase gráfica 3); en tanto, el endeudamiento por concepto de vivienda se mantiene relativamente constante a pesar de la gran promoción al sector inmobiliario, y el endeudamiento por consumo incrementa sin lograr montos significativos en el total.

Un aspecto relevante de este análisis son las fuentes del endeudamiento según destino de gasto, véase cuadro 2; donde se observa que las fuentes de endeudamiento del consumo son el crédito bancario y las tarjetas no bancarias, las cuales no representan fuertes movimientos, con excepción del periodo posterior a la crisis de 1994. El crédito bancario a la vivienda permanece relativamente constante (también con excepción del periodo 1995-2000), observándose un aumento sustancial de recursos provenientes de otras fuentes 


\begin{tabular}{|c|c|c|c|c|c|c|c|c|}
\hline & \multicolumn{3}{|c|}{ Credito Bancario } & \multicolumn{5}{|c|}{ No bancario } \\
\hline & Consumo & Vivienda & $\begin{array}{l}\text { E y Pf } \\
\text { c/AE }\end{array}$ & $\begin{array}{l}\text { TNB } \\
\text { Cons1 }\end{array}$ & $\begin{array}{c}\text { OFA } \\
\text { Vivienda } 2\end{array}$ & $\begin{array}{c}\text { E y PF IF } \\
\text { del País }\end{array}$ & $\begin{array}{l}\text { E y PF } \\
\text { OFA } 3\end{array}$ & $\begin{array}{l}\text { EyPE } \\
\text { F.Ext }\end{array}$ \\
\hline $1994 / 2013$ & 6.6 & 8.1 & 22.3 & 3.4 & 15.5 & 2.4 & 21.5 & 20.19 \\
\hline $1995-2000$ & 2.3 & 13.2 & 33.7 & 0.9 & 8.4 & 3.0 & 16.1 & 22.10 \\
\hline $2001 / 2007$ & 8.1 & 6.8 & 18.3 & 3.0 & 17.8 & 2.9 & 22.0 & 21.03 \\
\hline 2008-2010 & 6.4 & 7.9 & 20.7 & 4.9 & 17.7 & 1.8 & 22.1 & 18.52 \\
\hline $2011 / 2013$ & 7.1 & 7.2 & 20.9 & 4.5 & 15.9 & 1.5 & 23.7 & 19.28 \\
\hline
\end{tabular}

Fuente: Cálculo del autor, con base en las estadísticas del Banco de México, Cuadro -CF 88, Financiamiento al sector privado del pais y otras fuentes principales, metodologuia, 2003.

Ey PF c/AE: Empresas y Personas Físicas con actividad empresarial.

IF: intermediarios financieros.

E y PF c/AE: Empresas y Personas Físicas con actividad empresarial, otras fuentes alternativas.

$1 /$ Se refiere a tarjetas de crédito no bancarias relacionadas al credito del consumo.

2/ Se refiere a la cartera de crédito a la vivienda vigente y vencida del Instituto del Fondo Nacional de la Vivienda para los Trabajadores (INFONAVIT), y a la cartera hipotecaria vigente y vencida del Fondo de la Vivienda del issste (FOvissste). Fuente: Comisión Nacional Bancaria y de Valores. Se conjunto con el financiamiento de intermediarios financierios no bancarios.

3/ Corresponde al pasivo interno no bancario de las empresas que cotizan en la BMV y la emisión interna de instrumentos de deuda de empresas que no cotizan en BMV.

* Se calcula la participación de las fuentes por tipo de gasto con base en el promedio del total de endeudamiento.

Fuente: elaboración propia con base en

Disponible en: http://portafoliodeinformacion.cnbv.gob.mx/

alternativas (FOvissste e INFONAVIT), lo cual, implicó que la banca cedió la dominación de dicha actividad. A su vez, la deuda bancaria hacia las empresas y personas físicas con actividad empresarial se mantiene relativamente inalterada, mientras que las otras fuentes alternativas de financiamiento provenientes del país hacia las empresas y las personas físicas con actividad empresarial corresponde al pasivo interno no bancario de las empresas que cotizan en la BMV y la emisión interna de instrumentos de deuda de empresas que no cotizan en BMv logran un nivel equivalente a la deuda bancaria, lo cual, refleja la presencia de las grandes empresas trasnacionales de México que se financian con títulos de deuda del mercado dinero y de capital. El financiamiento externo representó otro $20 \%$ del promedio total de financiamiento, el cual, se ha mantenido relativamente inalterado, observándose una leve reducción en los años posteriores a la crisis de 2008.

Un quinto elemento es determinar el comportamiento del financiamiento al consumo y de la inversión, en tanto, el primero tiene una gran participación 
Economía Informa núm. 387 julio - agosto • 2014 " "

en el producto y en el tamaño del mercado interno; mientras el segundo es un indicador de la capacidad instalada, la cual, debería ser un gasto central en el contexto de una economía que se especializa en exportaciones, especialmente de manufacturas con altos contenidos tecnológicos, como es el caso de México, y excedentes en insumos productivos.

\section{Gráfica 4}

Evolución del consumo con respecto al PIB, financiamiento al consumo y tasa implícita de la cartera de créditos al consumo

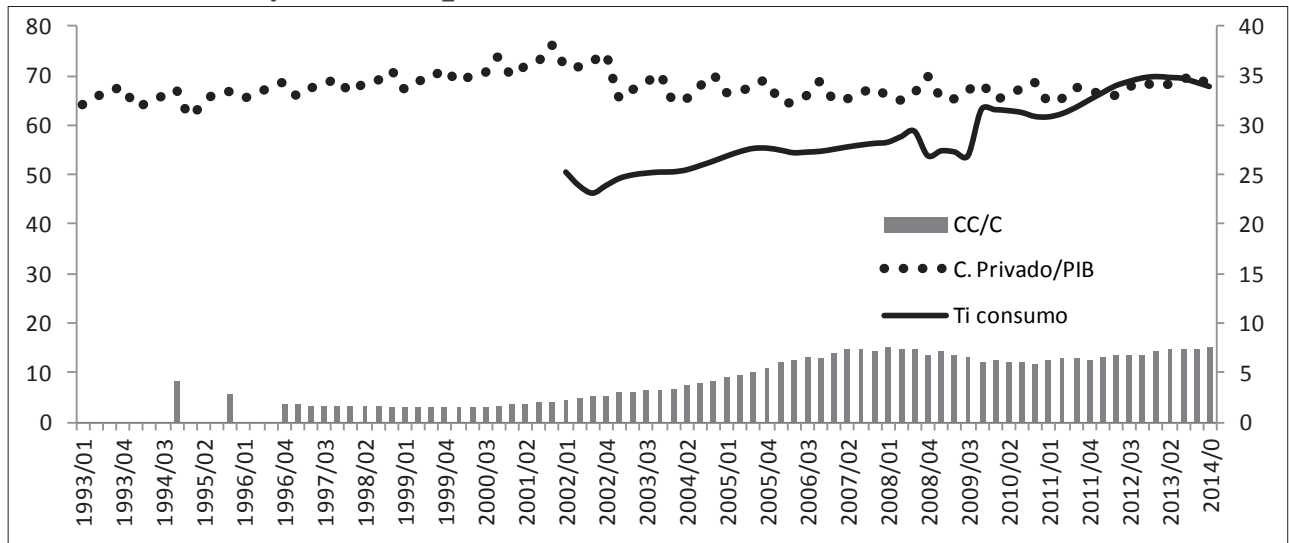

CC: Crédito al consumo en relacion al volumen del consumo (C).

C: C.Privado/PIB: Coeficiente del consumo privado con respecto al producto.

Ti consumo: tasa implicta de interés de los créditos al consumo.

Disponibe en: http://www.inegi.org.mx/sistemas/bie/, Producto interno bruto trimestral, base 2008 , Series originales, Valores a precios de 2008, Producto interno bruto, a precios de mercado (Periodicidad:Trimestral).

http://portafoliodeinformacion.cnbv.gob.mx/, Banca Múltiple, Información de la situación financiera, Indicadores financieros: históricos (series desde diciembre 2000).

http://www.banxico.org.mx/estadisticas/index.html, Financiamiento e información financiera de intermediarios financieros, Financiamiento Total al Sector Privado no Financiero.

La participación del consumo en el producto interno bruto ha presentado una tendencia descendente en la primera década del siglo xx. En el periodo observado los valores oscilan alrededor de $65 \%$, lo cual coincide con un aumento del endeudamiento de las familias por concepto de consumo. Ello está acompañado de un incremento de la tasas implícitas de créditos a la familias por concepto de consumo (véase gráfica 4), la cual, como se anotó anteriormente no responde a las variaciones de la tasa de referencia ni aumentos de riesgos. La relación de las tres variables indicaría que una política monetaria 
laxa no afecta al gasto del consumo privado y pese a su incremento, sube el financiamiento por ese concepto.

Finalmente el coeficiente de la inversión se ha mantenido relativamente constante a lo largo de las dos décadas analizadas no obstante la extranjerización de la industria mexicana y la apertura a la inversión extranjera directa de la economía mexicana y, especialmente a la luz del incremento de las exportaciones manufactureras de alto contenido tecnológico. El relativo estancamiento del gasto de la inversión con respecto al producto está acompañado de una menor tasa activa (o implícita) por concepto de créditos a las empresas, y paradójicamente se canaliza menos créditos a estos agentes. Ello indica que menores tasas de interés no activan la inversión de las empresas y la que realizan las personas físicas con actividad empresarial (véase gráfica 5).

\section{Gráfica 5}

Evolución del coeficiente de la inversión respecto al producto, endeudamiento a las empresas y personas con actividad física

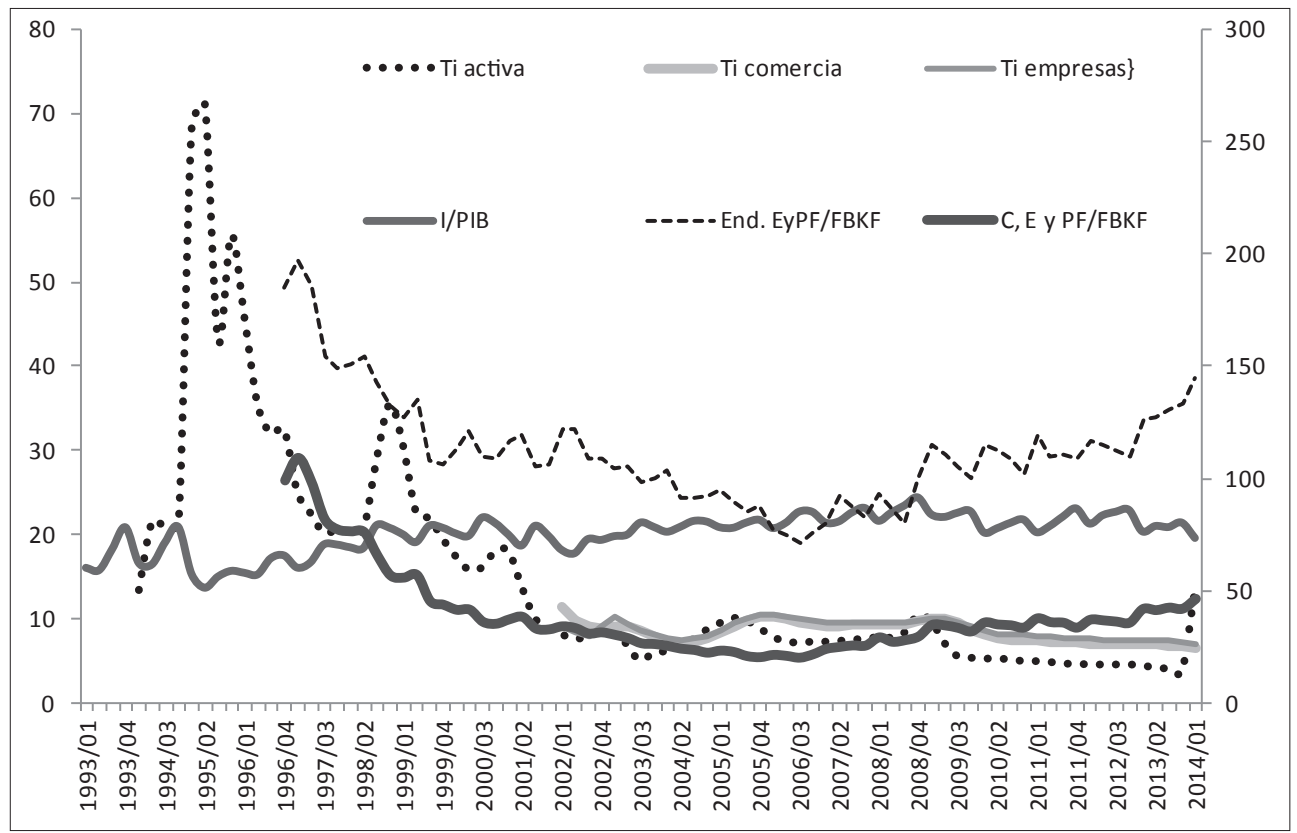

Ti: tasa de interés (eje izquierdo), I/PIB: coeficiente de inversión (eje izquierdo).

End. EyPF y C E y PF: endeudamiento a empresas y personas fisicas cion actividad empresarial y C. E y PF: créditos de empresas y personas físicas: FBKF: formacion bruta de capital fijo (eje derecho) Fuente: elaboración propia con base en: http://portafoliodeinformacion.cnbv.gob.mx/, Banca Múltiple, Información de la situación financiera, Indicadores financieros: históricos (series desde diciembre 2000). http://www.inegi.org.mx/sistemas/bie/ , Cuentas Nacionales, Oferta y utilización total de bienes y servicios, base 2008, Series originales, A precios constantes, Valores absolutos, Utilización de bienes y servicios, Consumo final, Formación bruta de capital fijo (Periocidad:Trimestral). http://www.banxico.org.mx/estadisticas/index.html, Financiamiento e información financiera de intermediarios financieros, Financiamiento Total al Sector Privado no Financiero. 
Economía Informa núm. 387 julio - agosto • 2014 - " " " " " " " " " -

\section{Conclusiones}

La tasa de interés es el principal instrumento de política monetaria, y la tasa de objetivo incide en las demás tasas de interés, aunque la tasa de Cetes de 91 días es una determinante importante a considerar en la determinación del Banco de México. Segundo, las variaciones de la tasa de interés, puede crear condiciones de astringencia o holgura, que tiene mayor impacto en la economía cuando se busca frenar la actividad económica. Por consiguiente, las reducciones en la tasa de interés tiene un bajo impacto en el crecimiento económico (aunque, entre otros objetivos, pueden ser el vehículo para reducir el peso de la deuda bancaria).

Tercero, no se encuentra una relación vigorosa entre menores tasas de interés, mayor crédito y crecimiento económico. La política monetaria laxa en condiciones de creciente "holgura" provocado por menores tasas de crecimiento del gasto interno no ha logrado dinamizar la economía. Desde la perspectiva de la teoría económica heterodoxa cambios en la tasa de interés no genere mayor crédito ni mayor actividad económica. El propio Keynes (1936), quien retomo de la teoría "clásica" la relación indirecta la relación entre la tasa de interés y la inversión, no respaldaría estas políticas, puesto que la principal limitación del crecimiento se genera por las ganancias esperadas, o sea la demanda agregada. Lo anteriormente señalado, porque el gasto de consumo está en función del ingreso, especialmente de remuneraciones de los trabajadores y el gasto de la inversión en función de las expectativas futuras. El gobierno estadounidense posterior a la crisis de 1929 captó este mensaje y, además de reducirlas tasas de interés, desplegó políticas de reactivación de la demanda, en un contexto de repudiación de la deuda especulativa generado por la inflación financiera de la década de los veinte del siglo pasado.

Entonces a qué responde las continuas reducciones de la tasa de interés de Banco de México. Aquí podríamos aventurar algunas respuestas. A nivel internacional ha tenido lugar una menor tasa de interés y la banca central se ha sintonizado con dicha tendencia. Segundo, el nivel de reservas internacionales se mantiene muy alto y no se espera que se reduzcan. Tercero no se espera que incremente la tasa interés de referencia estadounidense en el contexto de la estabilización de la política monetaria de dicho país. Cuarto y, quizás lo más relevante, la reducción de la tasa de referencia reduce la tasa de fondeo, sin disminuir la tasa implícita de la banca universal de la cartera de consumo y ello genera ganancias crecientes al sector bancario. Un efecto adicional es la 
reducción de la tasa de objetivo ser adecua a la caída de la tasa de Cetes de mayor plazo y reduce el costo de recaudación de ingresos por parte del gobierno.

Resumiendo, a partir de la mayor laxitud de la política monetaria de banco central no se puede esperar un mayor crecimiento económico ni una recuperación, ni siquiera temporal sino una reducción de costos para el sector gubernamental y el sector financiero endeudado o elevar los márgenes del sector bancario.

\section{Bibliografía}

Banco de México 2014, “Minuta número 28” Reunión de la Junta de Gobierno del Banco de México, con motivo de la decisión de política monetaria anunciada el 6 de junio de 2011, Véase en http://www.banxico.org.mx/informacion-para-laprensa/comunicados/politica-monetaria/minutas-de-las-decisiones-de-politica-monetaria/index.html.

Banco de Mexico, "Instrumentos de la Política Monetaria a través de un Objetivo Operacional de Tasas de Interés, http://www.banxico.org.mx/politica-monetaria-e-inflacion/material-de-referencia/intermedio/politicamonetaria/\%7B16DC84D2-A904-6373-28E3-29659E530FDB\%7D.pdf.

Bindseil, U. (2004), Monetary Policy Implementation, Theory, Past and Present,: Oxford University Press.

Blinder A. (1998), Central Banking in Theory and Practice, MIT Press,

Chick V. (2005), "Lost and found: some history of endogenous money in the twentieth century", The monetary theory of production: tradition and perspectives, Fontana, G. y R. Realfonzo (coordinadores), Basingstoke, Hampshire; New York, Palgrave Macmillan.

Domínguez, C. (2011) “Efectos de la concetración, eficiencia y barreras de entrada y salida en la rentabilidad de las actividades de la banca múltiple en Mexico, 20022007, Tesis de doctorado, Division de Estudios de la Posgrado de la Facultad de Economía, UNAM.

Fisher, I. (1933), “The Debt Deflation Theory of Great Depressions”, Econometrica vol. 1, no.1, pp.337-357.

Harris, L. (1981), Teoría monetaria, FCE, México, D.F.

Hüfner, F. (2004), "Foreign exchange intervention as a monetary policy instrument", Zew Economic Studies, 23, Physica-Verlag, Alemania.

Lavoie, M. (2004), "The new consensus on monetary Policy seen from a PostKeynesian perspective”, in Lavoie, M. and M. Seccareccia (eds), Central banking in the Modern World: Alternative perspectives, Cheltenham: Edward Elgar, pp. 15-34. 


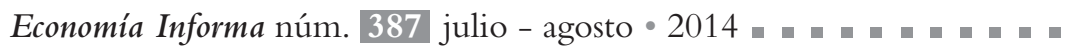

Levy, N. (2010a), La naturaleza de la tasa de interés y su impacto en el crecimiento económico ¿La política monetaria puede modificar la actividad económica? en Políticas Monetaria con elevado traspaso del tipo de cambio, la experiencia mexicana con metas de inflación Mántey G. y T. López (coordinadoras) Plaza y Valdez, México.

Levy N. (2010b), "Instituciones financieras para el desarrollo económico. Comparación del periodo de "sustitución de importaciones" y el "secundario exportador" en Cincuenta años de políticas financieras para el desarrollo, México, 1958-2008", Guadalupe Mántey y Noemi Levy (coordinadoras), Plaza y Váldez, México.

Mántey G. (2010), "Políticas financieras para el desarrollo en México ¿Qué hemos aprendido de 1958 a 2008" en Cincuenta años de políticas financieras para el desarrollo, México, 1958-2008. Guadalupe Mántey y Noemi Levy (coordinadoras), editorial Plaza y Váldez, México.

Modigliani, F. y L. Papademos (1975), “Targets for Monetary Policy in the Coming Year" Brookings Papers on Economic Activity, 141-165. The Brookings Institution.

Smithin J. (2007) "A real interest rate rule for monetary policy?, Journal of Post Keynesian Economics, Fall, vol. 30, núm. 1, pp. 101- 118.

Taylor, J. (1993), Discretion vs Policy Rules in Practice, Carnegie-Rochester Conference Series on Public Policy, December.

Toporowski J, 2005, Theories of financial Disturbance. An examination of critical theories of finance from Adam Smith to the present day, Edward Elgar.

Toporowski J, (2014) Credit and Crisis: From Marx to Minsky (en prensa).

Wicksell K. (1907) "The Influence of the Rate of Interest on Prices" The Economic Journal, Vol. 17, núm. 66 (Jun., 1907), pp. 213-220, www.jstor.org 